\title{
Surgical Flap and Graft Reconstruction Workshop for Dermatology Residents*
}

\author{
Brandon Goodwin, Richard Wagner \\ Department of Dermatology, The University of Texas Medical Branch, Galveston, TX, USA \\ Email: bpgoodwi@utmb.edu
}

Received 23 April 2015; accepted 5 June 2015; published 9 June 2015

Copyright $(02015$ by authors and Scientific Research Publishing Inc.

This work is licensed under the Creative Commons Attribution International License (CC BY). http://creativecommons.org/licenses/by/4.0/

(c) (i) Open Access

\section{Abstract}

Background: Traditional models for teaching surgical principles focus primarily on the apprenticeship theory; however there has been a trend in surgical education to certifying competency in a simulation environment prior to working with patients. Many surgical models emphasize learning the technical and manual dexterity skills necessary to be a surgeon, yet few focus on obtaining the theoretical and abstract skills needed for planning complex cutaneous surgical repairs with flaps and grafts. We developed and evaluated a novel surgical flaps and grafts workshop for residents through the Department of Dermatology. Methods: Participants received a 60 minute PowerPoint lecture focusing on the basic principles of cutaneous repair with flaps and grafts, with examples and explanation of each of the four main types of flaps and grafts. The participants then received nine photocopies of Mohs micrographic surgery defects with instructions to design three repairs, focusing on functional and aesthetic outcome. Hypothetical and actual repair designs were then discussed in an open forum format. Anonymous surveys administered to 11 dermatology residents assessed their knowledge level, confidence level, and likelihood of using flaps and grafts pre- and post-workshop using Likert scales. Overall experience was also assessed. A paired sample Wilcoxon Signed Rank Test was used for analysis, since the data was non-parametrically distributed. Results: There was a statistically significant increase in confidence performing flaps post workshop $(p=0.0469)$. There was also an increase in knowledge of flaps and grafts, confidence in planning flaps and grafts, and confidence in performing grafts post workshop, but these findings did not reach statistical significance. The workshop had no effect on expected future use of flaps and grafts. Conclusions: The surgical workshop is a novel simulation teaching tool for learning basic principles and design of flaps and grafts in cutaneous surgery.

\section{Keywords}

Surgical Skills Workshop, Flaps, Grafts, Dermatology, Resident Education

\footnotetext{
*Presented in part at the Dermatology Teachers Exchange Group, San Francisco, California, USA, March 23, 2015.
} 


\section{Introduction}

Traditional teaching models in surgical training revolve around the apprenticeship theory, "see one, do one, teach one" [1]. Most residents still obtain cutaneous surgery skills by assisting attending surgeons during surgical procedures, not within formal training environments [2]. Concerns over the adequacy of traditional surgical training have stemmed from a climate of decreasing clinical exposure during residency secondary to work hour restrictions and patient safety concerns [3]. In a recent study by Lee and colleagues of US dermatology residency programs, third year dermatology residents assume the role of the primary surgeon during $49 \%$ of flap and graft reconstruction cases and during 18\% of Mohs micrographic surgery cases [4]. As direct hands-on experience with flap and graft reconstruction of Mohs defects may be limited in some dermatology programs, exposure to learning through surgical apprenticeship is as well. Therefore it is imperative that new teaching models be developed to maximize learning these concepts.

The classic adult learner prefers learning activities that are experience oriented, self-directed, immediately applicable, internally motivated, and problem-centered [5]. A study assessing learning styles among US dermatology residents showed that active learning styles were significantly favored over passive teacher oriented learning styles overall and among male and female residents separately [5]. A surgical skills workshop incorporates many of the traditional active learning styles preferred by dermatology residents. Surgical skills workshops certify the competency of the learner in a stress free simulation environment thereby increasing confidence, knowledge and surgical ability prior to exposure to patients [6] [7]. While many surgical skills workshops have been developed to facilitate learning technical skills and manual dexterity essential in becoming a surgeon, few have been created to teach planning and design of complex repairs of surgical defects. We created a novel teaching tool for learning about and planning flap and graft reconstruction that is easy to use and assemble, inexpensive, incorporates a low-stress learning environment, and utilizes active learning.

\section{Background}

The Accreditation Council for Graduate Medical Education (ACGME) is the accrediting body for US post graduate medical training programs, including dermatology programs. According to the ACGME, a dermatology residency program must provide a clinical experience with significant exposure to Mohs micrographic surgery, and wound reconstruction, including flaps and grafts. Residents must be able to competently perform all surgical procedures considered essential for the area of practice including closures of surgical defects with attention to the patient outcome. Residents must also demonstrate knowledge of proper techniques for repairs of cutaneous surgical defects using flaps and grafts [8].

\section{Materials and Methods}

Eleven dermatology residents participated in the surgical flap and graft reconstruction workshop during the 2014-2015 academic year. The workshop started with an anonymous pre-workshop questionnaire (Appendix 1) followed by a 60-minute PowerPoint presentation that focused on the basic principles of flap and graft reconstruction with specific examples and explanations of the main types of flaps and grafts [9]. Identified learning objectives included: identify and know the four main types of flaps (advancement, rotation, transposition, and interpolation flaps); understand how each of the four main types of flaps move; understand how/when flaps may be used in dermatological surgery; identify and know the four main types of grafts (full thickness, split thickness, composite, and free cartilage grafts); and understand how/when grafts may be used in dermatological surgery. After the presentation, the participants were given nine photocopies of Mohs micrographic surgery defects (cheek, forehead, dorsal hand, nose \{nasal sidewall-small/large, nasal tip\}, upper cutaneous lip, ear \{superior helix, lobule\}) with instructions (Appendix 2) to design three repairs for each defect, focusing on functional and aesthetic outcome for the patient. Participants had one week to design their repairs. No repair was considered "off limits", but the participants were expected to be able to defend why one repair was chosen over another. On follow up, participants met in an open forum format to discuss both the hypothetical repairs (Figure 1) designed by residents and the actual repair performed following Mohs micrographic surgery. The attending Mohs surgeon was present to facilitate the dialogue and provide guidance and expertise when discussing repair options. The workshop concluded with an anonymous post-workshop questionnaire (Appendix 3). Internal departmental educational activities are not subject to Institutional Review Board review. 

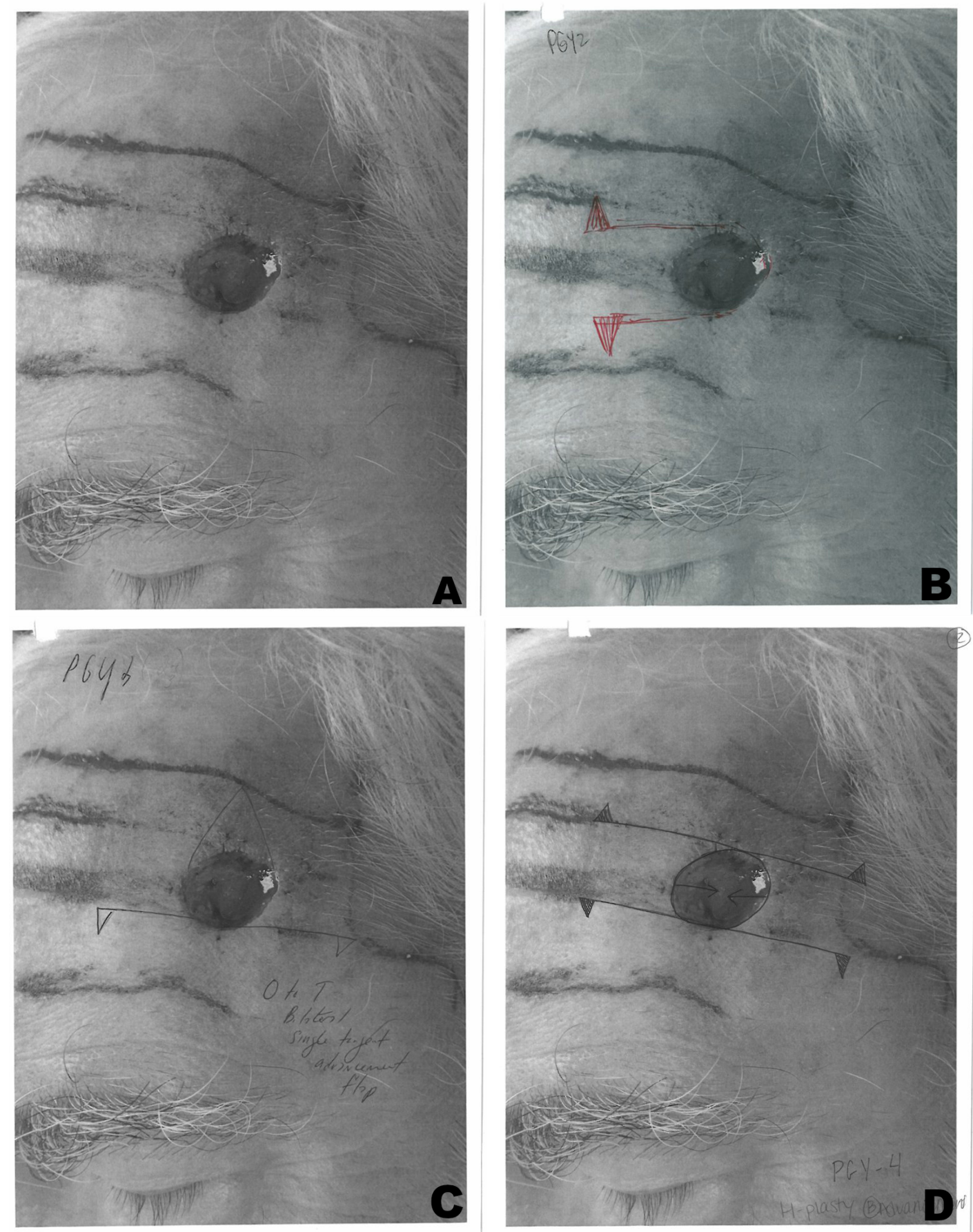

Figure 1. MMS forehead defect and repairs designed by different post graduate year (PGY) participants. (A) Mohs micrographic surgery forehead defect; (B) post graduate year 2 repair design; unilateral double tangent advancement flap; (C) post graduate year 3 repair design; O to T bilateral single tangent advancement flap; (D) post graduate year 4 repair design; Hplasty bilateral double tangent advancement flap.

In the anonymous surveys administered for the pre and post skills workshop, respondents were asked to assess their overall knowledge of flap and graft repairs, confidence level in both planning and performing flap and graft repairs, if the stated learning objectives were met during the workshop, if the workshop was a good educational experience and the likelihood of flap and graft use in future practice using Likert scales. Likert scale responses of four or higher ( 4 = somewhat agree, $5=$ completely agree) were considered "positive", and a response of 
three or lower ( 3 = neutral, 2 = somewhat disagree, 1 = completely disagree) were considered "negative" in evaluating participant responses for assessment of the workshop obtaining its learning objectives and its overall educational experience. The participants were also asked to assess overall surgical experience with flaps and graft repair using yes/no response questions prior to the workshop. A paired sample Wilcoxon Signed Rank non-parametric test was used to compare the medians for pre- vs. post-workshop survey questions. This test was used to account for non-normal distribution of the data using SAS for window software, version 9.3.

\section{Results}

Ninety one percent (10/11) of respondents had witnessed a flap reconstruction and 73\% (8/11) of respondents had performed a flap reconstruction prior to the surgical workshop. The percentage of flap repairs witnessed and performed increased with post graduate year of training. Of the 73\% (8/11) of respondents who had performed a flap repair in the past, 50\% (4/8) had performed less than five repairs and 50\% (4/8) had performed greater than 10 repairs. The number of flap repairs increased with post graduate year training, with $100 \%$ (3/3) of PGY-4 residents performing greater than 10 flap repairs.

Ninety one percent (10/11) of respondents had witnessed a graft reconstruction and 55\% (6/11) of respondents had performed a graft reconstruction prior to the surgical workshop. The percentage of graft repairs witnessed did not increase with post graduate year level, while the percent performed did increase with post graduate year of training. Of the $55 \%(6 / 11)$ of respondents who had performed a graft repair in the past, 50\% (3/6) had performed less than five repairs and 50\% (3/6) had performed greater than 10 repairs. The number of graft repairs increased with post graduate year training, with 100\% (3/3) of PGY-4 residents performing greater than 10 graft repairs.

Results showed a statistically significant change in confidence with performing flap reconstruction $(\mathrm{p}=$ 0.0469) post workshop. There was an increase in knowledge of flaps and grafts, confidence in planning flaps and grafts, and confidence in performing grafts post workshop. The workshop had no effect on the likelihood of participant's future use of flap and graft reconstruction. However this data failed to reach statistical significance, most likely due to a small sample size $(\mathrm{n}=11)$.

Positive response rates for each of the learning objectives are as follows: identify and know the 4 main types of flaps (100\%, $11 / 11$ positive); understand how each of the four main types of flaps move (73\%, 8/11 positive); understand how/when flaps may be used in dermatological surgery (82\%, 9/11 positive); identify and know the four main types of grafts (100\%, 11/11 positive); and understand how/when grafts may be used in dermatological surgery (91\%, 10/11 positive). Eighty two percent (9/11) of participants thought the flap and graft surgical workshop was a good educational experience.

\section{Discussion}

There was an increase in participant knowledge of flaps and grafts, confidence in planning flaps and grafts, and confidence in performing flaps and grafts post workshop, but only confidence in performance of flaps reached statistical significance. Research supports our above finding that surgical workshops increase participants' ability to perform procedural skills and their confidence [1] [2] [6]. Overall, the majority of participants found the workshop to be useful and a good educational experience for learning about flap and graft repairs.

Limitations of our study include a small number of participants $(n=11)$, focusing only on surgical theory while disregarding actual "hands-on" training for flap and graft reconstruction, and poor quality photocopies with lack of defect measurements, which may have influenced repair decisions. In the future, hands on training with surgical simulation models can be added to apply the concepts learned with the current flap and graft workshop.

\section{Conclusion}

Since dermatology residents must demonstrate knowledge of proper techniques for repairs of cutaneous surgical defects using flaps and grafts prior to graduation, an interactive surgical workshop focusing on the planning of defect repairs using flap and graft reconstruction may be a beneficial addition to a surgical curriculum [8]. As dermatology residents tend to prefer active teaching styles, implementing active teaching styles will often lead to a more enjoyable educational experience [5]. We present the surgical flap and graft reconstruction workshop 
as a novel teaching tool for learning flaps and grafts that is easy to use and assemble, inexpensive, incorporates a low stress learning environment, and utilizes active learning.

\section{References}

[1] Banks, E., et al. (2006) A Surgical Skills Laboratory Improves Residents' Knowledge and Performance of Episiotomy Repair. American Journal of Obstetrics and Gynecology, 195, 1463-1467. http://dx.doi.org/10.1016/j.ajog.2006.05.041

[2] Altinyazar, H.C., Hosnuter, M., Unalacak, M., Koca, R. and Babuccu, O. (2003) A Training Model for Cutaneous Surgery. Dermatological Surgery, 29, 1122-1124.

[3] Nicholas, L., Toren, K., Bingham, J. and Marquart, J. (2013) Simulation in Dermatologic Surgery: A New Paradigm in Training. Dermatological Surgery, 39, 76-81. http://dx.doi.org/10.1111/dsu.12032

[4] Lee, E., Nehal, K., Dusza, S., Hale, E. and Levine, V. (2011) Procedural Dermatology Training during Dermatology Residency: A Survey of Third-Year Dermatology Residents. Journal of the American Academy of Dermatology, 64, 475-483. http://dx.doi.org/10.1016/j.jaad.2010.05.044

[5] Stratman, E., Vogel, C., Rick, S. and Mukesh, B. (2008) Analysis of Dermatology Resident Self-Reported Successful Learning Styles and Implications for Core Competency Curriculum Development. Medical Teacher, 30, 420-425. http://dx.doi.org/10.1080/01421590801946988

[6] Adams, C., Marquart, J., Nicholas, L., Sperling, L. and Meyerle, J. (2014) Survey of Medical Student Preference for Simulation Models for Basic Dermatologic Surgery Skills: Simulation Platforms in Medical Education. Dermatological Surgery, 40, 427-435. http://dx.doi.org/10.1111/dsu.12445

[7] Ghiabi, E. and Taylor, L. (2010) Teaching Methods and Surgical Training in North American Graduate Periodontics Programs: Exploring the Landscape. Journal of Dental Education, 74, 618-627.

[8] (2014) ACGME (Accreditation Council for Graduate Medical Education). http://www.acgme.org/acgmeweb/Portals/0/PFAssets/ProgramRequirements/080_dermatology_07012014_u06152014. pdf

[9] Bolognia, J.L., Jorizzo, J.L. and Schaffer, J.V., Eds (2012) Bolognia Textbook of Dermatology. 3rd Edition, Mosby Elsevier Publishing, Spain, Chapters 147-148. 


\section{Appendix 1. Anonymous Pre Flap/Graft Surgical Workshop Survey}

Year (PGY 2, PGY 3, PGY 4):

I have witnessed a surgical flap repair in the past

(1) Yes

(2) No

I have performed a surgical flap repair in the past

(1) Yes

(2) No

I have witnessed a surgical graft repair in the past

(3) Yes

(4) No

I have performed a surgical graft repair in the past

(1) Yes

(2) No

Regarding the number of surgical flap repair opportunities (performed and witnessed), I have participated in:

(1) $<5$

(2) $5-10$

(3) $>10$

Regarding the number of surgical graft repair opportunities (performed and witnessed), I have participated in:

(1) $<5$

(2) $5-10$

(3) $>10$

My surgical flap repair knowledge prior to participation in the flap/graft surgical workshop

$\begin{array}{lllll}1 & 2 & 3 & 4 & 5\end{array}$

( 1 = no experience, 2 = below average, 3 = average, 4 = above average, 5 = excellent)

My surgical graft repair knowledge prior to participation in the flap/graft surgical workshop

$\begin{array}{lllll}1 & 2 & 3 & 4 & 5\end{array}$

( 1 = no experience, 2 = below average, 3 = average, 4 = above average, 5 = excellent)

I feel confident planning surgical defect repairs with flap procedures

$\begin{array}{lllll}1 & 2 & 3 & 4 & 5\end{array}$

( 1 = completely disagree, 2 = somewhat disagree, $3=$ neutral, $4=$ somewhat agree, $5=$ completely agree)

I feel confident planning surgical defect repairs with graft procedures

$\begin{array}{lllll}1 & 2 & 3 & 4 & 5\end{array}$

( 1 = completely disagree, 2 = somewhat disagree, $3=$ neutral, $4=$ somewhat agree, $5=$ completely agree)

I feel confident performing surgical repairs with flap procedures

$\begin{array}{lllll}1 & 2 & 3 & 4 & 5\end{array}$

( 1 = completely disagree, 2 = somewhat disagree, $3=$ neutral, $4=$ somewhat agree, 5 = completely agree)

I feel confident performing surgical repairs with graft procedures

$\begin{array}{lllll}1 & 2 & 3 & 4 & 5\end{array}$

( 1 = completely disagree, 2 = somewhat disagree, $3=$ neutral, $4=$ somewhat agree, $5=$ completely agree $)$

I am likely to use surgical flaps and graft repairs in the future

$\begin{array}{lllll}1 & 2 & 3 & 4 & 5\end{array}$

( 1 = completely disagree, 2 = somewhat disagree, 3 = neutral, $4=$ somewhat agree, $5=$ completely agree) 


\section{Appendix 2. Anonymous Flap/Graft Surgical Workshop Instructions}

- You are given nine Mohs micrographic surgery (MMS) defects with negative margins in need of repair. -Cheek, forehead, dorsal hand, nose (nasal sidewall-small/large, nasal tip), upper cutaneous lip, ear (superior helix, lobule)

- Develop three different repair options for each MMS defect by drawing your planned repair on the handout.

- All methods for repair may be used (primary repair, split thickness skin graft, full thickness skin graft, advancement flaps, rotational flaps, transposition flaps, interpolation flaps, etc...).

-All repairs must keep in mind both functional and aesthetic concerns for the patient (free margins, cosmetic subunits, relaxed skin tension lines, tissue laxity, surgical danger zones, etc...).

- After completing your three repairs for the MMS defect place your post graduate year level at the top of each handout. Avoid any other identifying factors.

-We will discuss repair options for each of the defects in one week with an open forum discussion.

- Remember, there is no one "right” way to repair a defect, but be prepared to discuss why one repair option was chosen over the other.

- At the end of the discussion, the anonymous MMS defect/repairs will be collected.

\section{Appendix 3. Anonymous Post Flap/Graft Surgical Workshop Survey}

Year (PGY 2, PGY 3, PGY 4):

My surgical flap repair knowledge after participation in the flap/graft surgical workshop

$\begin{array}{lllll}1 & 2 & 3 & 4 & 5\end{array}$

( 1 = no experience, 2 = below average, 3 = average, 4 = above average, 5 = excellent)

My surgical graft repair knowledge after participation in the flap/graft surgical workshop

$\begin{array}{lllll}1 & 2 & 3 & 4 & 5\end{array}$

( 1 = no experience, 2 = below average, 3 = average, 4 = above average, 5 = excellent)

I feel confident planning surgical defect repairs with flap procedures

$\begin{array}{lllll}1 & 2 & 3 & 4 & 5\end{array}$

(1 = completely disagree, 2 = somewhat disagree, 3 = neutral, $4=$ somewhat agree, $5=$ completely agree)

I feel confident planning surgical defect repairs with graft procedures

$\begin{array}{lllll}1 & 2 & 3 & 4 & 5\end{array}$

( 1 = completely disagree, 2 = somewhat disagree, $3=$ neutral, $4=$ somewhat agree, 5 = completely agree)

I feel confident performing surgical repairs with flap procedures

$\begin{array}{lllll}1 & 2 & 3 & 4 & 5\end{array}$

( 1 = completely disagree, 2 = somewhat disagree, $3=$ neutral, $4=$ somewhat agree, $5=$ completely agree)

I feel confident performing surgical repairs with graft procedures

$\begin{array}{lllll}1 & 2 & 3 & 4 & 5\end{array}$

( 1 = completely disagree, 2 = somewhat disagree, $3=$ neutral, $4=$ somewhat agree, $5=$ completely agree $)$ 
I am likely to use surgical flaps and graft repairs in the future

$\begin{array}{lllll}1 & 2 & 3 & 4 & 5\end{array}$

( 1 = completely disagree, 2 = somewhat disagree, 3 = neutral, 4 = somewhat agree, 5 = completely agree)

The surgical flap and grafts workshop was a good educational experience

$\begin{array}{lllll}1 & 2 & 3 & 4 & 5\end{array}$

( 1 = completely disagree, 2 = somewhat disagree, 3 = neutral, 4 = somewhat agree, 5 = completely agree $)$

Were the following learning objectives met during the presentation and workshop?

1. Identify and know the 4 main types of flaps $\quad \begin{array}{llllll}1 & 2 & 3 & 4 & 5\end{array}$

2. Understand how each of the 4 main types of flaps move $\quad \begin{array}{llllll}1 & 2 & 3 & 4 & 5\end{array}$

3. Understand how/when flaps may be used in dermatological surgery $\begin{array}{llllll}1 & 2 & 3 & 4 & 5\end{array}$

4. Identify and know the 4 main types of grafts $\quad \begin{array}{llllll} & 1 & 2 & 3 & 4 & 5\end{array}$

5. Understand how/when grafts may be used in dermatological surgery $\begin{array}{llllll}1 & 2 & 3 & 4 & 5\end{array}$

( 1 = completely disagree, 2 = somewhat disagree, $3=$ neutral, $4=$ somewhat agree, $5=$ completely agree)

Are there any changes you would recommend for future flap/graft workshops:

Additional comments: 